\title{
DESEMPENHO DE ESCOLARES COM E SEM DIFICULDADES \\ DE APRENDIZAGEM DE ENSINO PARTICULAR EM HABILIDADE FONOLÓGICA, NOMEAÇÃO RÁPIDA, LEITURA E ESCRITA
}

\author{
Performance of students with and without learning difficulties \\ in phonological awareness, rapid naming, reading and writing \\ from the private education
}

\author{
Simone Aparecida Capellini ${ }^{(1)}$, Talita Laura Braz Capano Conrado (2)
}

\begin{abstract}
RESUMO
Objetivos: caracterizar e comparar o desempenho de escolares com e sem dificuldades de aprendizagem no ensino particular em habilidades fonológicas, nomeação rápida, leitura e escrita. Métodos: participaram desse estudo 60 escolares de $2^{-}$a $4^{\underline{a}}$ séries de escola de ensino particular, distribuídos em 6 grupos, sendo cada grupo composto por 10 escolares, sendo 3 grupos de escolares com dificuldades de aprendizagem e 3 grupos de escolares sem dificuldades de aprendizagem. Como procedimentos, foram realizadas a prova de nomeação automática rápida, a de consciência fonológica e a prova de leitura oral e escrita sob ditado. Resultados: os resultados desse estudo evidenciaram desempenho superior dos escolares sem dificuldades de aprendizagem em relação àqueles com dificuldades. Os escolares com dificuldades de aprendizagem apresentaram maior relação velocidade/ tempo em tarefas de nomeação e, consequentemente, desempenho inferior em tarefas de consciência fonológica e leitura e escrita de palavras isoladas quando comparados aos sem dificuldades de aprendizagem. Conclusão: os escolares com dificuldades de aprendizagem apresentaram comprometimento na relação entre as capacidades de nomeação e automatização dos estímulos apresentados com a capacidade de acesso lexical, discriminação visual, frequência de uso dos estímulos e competição para a apresentação do menor tempo possível na nomeação dos códigos necessários para o estabelecimento do mecanismo de conversão fonema-grafema, exigido para a realização da leitura e escrita em um sistema alfabético como o português.
\end{abstract}

DESCRITORES: Leitura; Transtornos de Aprendizagem; Educação

\section{INTRODUÇÃO}

As dificuldades de aprendizagem podem ser entendidas como obstáculos ou barreiras encontradas por alunos durante o período de escolarização,

(1) Fonoaudióloga; Docente do Departamento de Fonoaudiologia e da Pós-Graduação em Educação da Faculdade de Filosofia e Ciências da Universidade Estadual Paulista, UNESP, Marília, SP; Doutora em Ciências Médicas pela Faculdade de Ciências Médicas, Campinas, SP.

(2) Fonoaudióloga; Aluna especial do Programa de Pós-graduação em Educação da Faculdade de Filosofia e Ciências da Universidade Estadual Paulista, UNESP, Marilia, SP.

Conflito de interesse: inexistente referentes à captação ou à assimilação dos conteúdos propostos. Essas dificuldades podem ser duradouras ou passageiras e mais ou menos intensas. Podem levar alunos ao abandono da escola, à reprovação, ao baixo rendimento, ao atraso no tempo de aprendizagem ou mesmo à necessidade de ajuda especializada ${ }^{1,2}$.

Estudo realizado em $1994{ }^{3}$ apontou que apenas $43 \%$ das crianças que ingressam no sistema educacional terminam o ensino fundamental e, dentre estas, apenas $13 \%$ não lidam com repetências, ou seja, conseguem chegar à oitava série na idade esperada. Mais recentemente, dados divulgados pelo Instituto Nacional de Educação e Pesquisa ${ }^{4,5}$ 
revelaram que $59 \%$ dos alunos do ensino básico, no país, apresentam rendimento considerado crítico ou muito crítico e que a taxa de distorção sérieidade atinge $39 \%$ do total de alunos avaliados. No Brasil, não há estimativa sobre prevalência das dificuldades de aprendizagem pelo fato desta categoria diagnóstica não se situar no sistema educacional. Entretanto, a inaptidão para a leitura afeta de 2 a $8 \%$ das crianças em escolas de ensino fundamental $^{6}$.

Nesta realidade educacional, existe ainda uma lacuna entre este pré-diagnóstico que é realizado pelos professores de forma competente e assertiva e o uso do mesmo não como rótulo, mas como um auxílio para o professor realizar um planejamento de ensino mais adequado a partir do levantamento das dificuldades de aprendizagem para a minimização das alterações de leitura, escrita e cálculomatemático destas crianças ${ }^{7}$.

O estudo acima citado comparou o desempenho de escolares em relação à avaliação formal e à opinião de professores. As médias do grupo definido pelos professores como apresentando desempenho inferior foram abaixo do esperado para o grupo classe, evidenciando o fato de que a opinião daqueles foi fundamental para a identificação dos problemas relacionados à escolaridade.

Estudos recentes demonstram que crianças com dificuldades de aprendizagem podem apresentar alterações em habilidades fonológicas e no acesso ao léxico mental decorrentes de modificações em diferentes níveis do processamento da informação. Há três décadas, pesquisas demonstram que a velocidade de nomeação de estímulos encontra-se intimamente relacionada à velocidade de acesso à memória de curto prazo e à nomeação fonológica, que influenciam diretamente no desenvolvimento da leitura e escrita ${ }^{8-12}$.

As crianças com dificuldades de leitura apresentam fluência de leitura alterada e problemas com a compreensão de leitura em decorrência de alterações de percepção fonológica e baixa capacidade de armazenamento de informação na memória de trabalho ${ }^{13}$.

Os mecanismos componentes do processamento da linguagem estão presentes de forma subjacente ao desenvolvimento da consciência fonológica. Esses componentes, ou seja, a memória fonológica de trabalho e o acesso fonológico ao léxico mental permitem o processamento e a organização da linguagem. Da mesma forma, eles são solicitados pelo componente executivo central na realização de qualquer tarefa, inclusive nas de consciência fonológica e associação fonema-grafema ${ }^{14}$.

Com base no exposto acima, este estudo teve por objetivos caracterizar e comparar o desempe- nho de escolares com e sem dificuldades de aprendizagem, no ensino particular, referente às habilidades fonológicas, nomeação rápida, leitura e escrita.

\section{MÉTODOS}

Participaram deste estudo transversal 60 escolares da $2^{\mathrm{a}}$ a $4^{\mathrm{a}}$ séries de escola particular do município de Marília-SP, sendo 32 (53\%) do sexo masculino e 28 (47\%) do feminino, com faixa etária variando de 8 anos e 3 meses a 10 anos e 4 meses, distribuídos nos seguintes grupos:

Grupo I (GI): composto por 10 escolares com dificuldade de aprendizagem, da $2^{\mathrm{a}}$ série;

Grupo II (GII): composto por 10 escolares com dificuldade de aprendizagem, da $3^{\mathrm{a}}$ série;

Grupo III (GIII): composto por 10 escolares com dificuldade de aprendizagem, da $4^{a}$ série;

Grupo IV (GIV): composto por 10 escolares sem dificuldade de aprendizagem, da $2^{\mathrm{a}}$ série;

Grupo V (GV): composto por 10 escolares sem dificuldade de aprendizagem, da $3^{\mathrm{a}}$ série;

Grupo VI (GVI): composto por 10 escolares sem dificuldade de aprendizagem, da $4^{a}$ série.

Os escolares foram selecionados pelos professores com base no desempenho acadêmico em dois bimestres consecutivos, sendo considerados escolares com dificuldades de aprendizagem aqueles que apresentavam desempenho insuficiente e sem dificuldades de aprendizagem aqueles que apresentavam desempenho suficiente.

A distribuição dos escolares nos grupos acima apresentados ocorreu mediante orientação estatística, o que possibilitou melhor tratamento e comparação dos achados que serão apresentados neste estudo.

Para a realização do presente estudo, foram utilizados os seguintes procedimentos:

A) Termo de Consentimento Pós-Informado: Conforme resolução do Conselho Nacional de Saúde CNS 196/96, anteriormente ao início das avaliações, os pais ou responsáveis dos escolares selecionados assinaram o Termo de Consentimento Pós-Informado para autorização da realização do estudo.

B) Prova de Nomeação Automática Rápida (RAN): Os escolares selecionados foram submetidos à aplicação da Prova de Nomeação Automática Rápida ${ }^{15}$, adaptada por Ferreira et al. ${ }^{16}$, composta pelos subtestes de nomeação de cores, dígitos, letras e objetos. Os subtestes compreendem 5 estímulos diferentes, que se alternam formando ao todo dez linhas sequenciais, num total de cinquenta estímulos. 
Antes do início da prova, os escolares receberam a orientação de nomear os estímulos com a maior velocidade possível, sem erros. Os escolares foram orientados de que o tempo seria cronometrado (cronômetro KK- 1039) a partir do momento do sinal inicial do teste.

C) Prova de Consciência Fonológica: O procedimento utilizado foi a Prova de Consciência Fonológica ${ }^{17}$, constituída de 10 subtestes, com 4 itens cada, que se referem à verificação das habilidades fonêmicas e silábicas de síntese, segmentação, manipulação e transposição; supra-fonêmicas, como rima e aliteração.

D) Prova de Leitura e Escrita: Os procedimentos utilizados foram a leitura oral e a escrita por meio de ditado ${ }^{18}$, compreendendo-se 2 sub-listas de 48 palavras reais e 48 inventadas, totalizando $96 \mathrm{em}$ cada categoria. As mesmas listas foram utilizadas para as tarefas de leitura oral e escrita por meio de ditado, tendo sido analisadas de forma quantitativa, ou seja, por frequência de erros.

Os procedimentos deste estudo foram realizados no Centro de Estudos da Educação e Saúde CEES/FFC/Unesp - Marília, pelos autores em horário contrário ao da escola. Foram aplicados em uma sessão individual de 1 hora e 30 minutos. As respostas dos escolares à testagem foram registrados em folhas respostas.

Este trabalho foi realizado após aprovação do Comitê de Ética em Pesquisa da Faculdade de Filosofia e Ciências da Universidade Estadual Paulista FFC/UNESP/Marília - SP, sob o protocolo de número 2812/2003.

Os resultados deste estudo foram analisados estatisticamente visando comparar o desempenho quanto à habilidade fonológica, à nomeação automática rápida e à habilidade de leitura e escrita entre escolares com e sem dificuldades de aprendizagem. Para a análise estatística inter-grupos, foi utilizado o Teste Mann-Whitney e, para análise estatística intra-grupos, foi utilizado o Teste Wilcoxon. Para a realização da análise estatística e obtenção dos resultados, foi utilizado o programa SPSS (Statistical Package for Social Sciences), em sua versão 10.0 .

\section{RESULTADOS}

Na Tabela 1, apresenta-se a média do desempenho dos grupos no Teste de Nomeação Rápida Automática (RAN) na Prova de Consciência Fonológica (PCF) e Prova de Leitura Oral e Escrita sob Ditado.

Quando comparados o desempenho dos grupos com e sem dificuldades de aprendizagem, verifica-se na Tabela 2 que houve diferenças estatisticamente significante quando se compara o Gll e o GV para nomeação de letras, números e objetos e para os escolares dos GIII e GVI na nomeação de cores, letras e objetos. Quanto a habilidades fonológicas, observam-se diferenças significantes quando comparados os desempenhos dos escolares dos $\mathrm{Gl}$ e GIV nos subtestes de manipulação silábica e manipulação fonêmica; GIl e GV nos subtestes de rima, síntese fonêmica, manipulação fonêmica, transposição fonêmica e escore total.

Ainda na Tabela 2 observam-se diferenças significantes quando comparados os desempenhos dos escolares dos GI e GIV, GII e GV e GIII e GVI na prova de leitura oral. Os GIII e GIV apresentaram diferenças estatisticamente significantes na categoria de palavras reais regulares de alta frequência, enquanto que os Gll e GV apresentaram diferenças estatisticamente significantes na categoria de palavras reais irregulares de baixa frequência. Os escolares dos GI e GIV, GIl e GV apresentaram diferenças estatisticamente significantes nas categorias de palavras reais de baixa e alta frequências regra e nas palavras inventadas regulares, irregulares e regra.

Quanto ao desempenho dos grupos na prova de escrita sob ditado, verifica-se que o GI e GIV apresentaram diferenças estatisticamente significantes em todas as categorias de palavras regulares, regra e irregulares de alta e baixa frequências, enquanto os Gll e GV apresentaram tal diferença nas palavras reais regulares de alta frequência, nas reais irregulares de baixa frequência, nas reais de alta e baixa frequências regra, nas inventadas regulares e regra. Os grupos GIII e GVI apresentaram diferenças estatisticamente significantes em todas as categorias, exceto nas palavras reais de alta frequência regra, nas palavras inventadas irregulares e regra.

A Tabela 3 apresenta a comparação intra-grupo das médias referentes ao desempenho dos escolares dos GI, GII, GIII, GIV, GV e GVI no Teste de Nomeação Rápida Automática (RAN) em relação aos sub testes de cores, letras, números e objetos. É possível verificar que ocorreram diferenças estatisticamente significantes para cores e letras, cores e números, letras e objetos e números e objetos em todos os grupos. Os escolares dos GI, GII e GV apresentaram esta diferença somente para a nomeação de letras e números.

Realizada a comparação intra-grupo das médias referentes ao desempenho dos grupos na Prova de Consciência Fonológica (PCF), em relação às habilidades fonêmicas, supra-fonêmicas e silábicas, verificou-se que ocorreram diferenças estatisticamente significantes para todos os grupos quando comparadas às habilidades fonêmicas e silábicas de síntese, segmentação e transposição. Quanto 
Tabela 1 - Distribuição das médias do desempenho dos escolares dos GI, GII, GIII, GIV, GV e GVI nos sub testes do RAN, PCF e Leitura Oral e Escrita sob ditado de palavras e pseudo-palavras

\begin{tabular}{|c|c|c|c|c|c|c|c|}
\hline \multirow{5}{*}{$\underset{\nwarrow}{\mathbf{x}}$} & Habilidades & GI & GII & GIII & GIV & GV & GVI \\
\hline & Cor & 75,7 & 56,3 & 66,9 & 60,9 & 44,7 & 43,5 \\
\hline & Letra & 54,5 & 37,7 & 35,5 & 36,9 & 24,6 & 25,3 \\
\hline & Números & 51,6 & 33,7 & 34,8 & 37,2 & 28,0 & 25,6 \\
\hline & Objetos & 88,6 & 70,4 & 74,0 & 74,9 & 59,4 & 50,8 \\
\hline \multirow{11}{*}{ 岕 } & Sis & 0 & 0 & 0,5 & 0 & 0 & 0 \\
\hline & SiF & 2,6 & 1,9 & 3,4 & 2,7 & 2,0 & 1,7 \\
\hline & Ses & 2,3 & 2,3 & 1,2 & 1,0 & 0,2 & 0 \\
\hline & SeF & 1,8 & 1,4 & 0,8 & 1,1 & 0 & 0,1 \\
\hline & Rim & 0 & 0 & 0 & 0 & 0,1 & 0 \\
\hline & Alit & 4,0 & 3,6 & 3,0 & 3,4 & 2,7 & 2,1 \\
\hline & ManS & 1,1 & 1,7 & 1,3 & 0,2 & 0,1 & 0 \\
\hline & ManF & 2,6 & 2,4 & 1,1 & 1,3 & 0,1 & 0 \\
\hline & TrS & 1,2 & 1,1 & 0,6 & 0,9 & 0,6 & 0 \\
\hline & $\operatorname{TrF}$ & 4,0 & 3,5 & 3,9 & 3,5 & 2,6 & 1,5 \\
\hline & ET & 20,1 & 18,2 & 15,8 & 14,1 & 8,8 & 5,4 \\
\hline \multirow{9}{*}{ 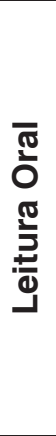 } & PRRAF & 8,7 & 5,7 & 3,3 & 3,0 & 0,5 & 1,3 \\
\hline & PRgAF & 6,3 & 5,2 & 2,0 & 2,1 & 0,4 & 0,4 \\
\hline & PRIrAF & 10,7 & 9,0 & 7,0 & 5,5 & 1,5 & 3,3 \\
\hline & PRRBF & 8,7 & 7,3 & 2,8 & 2,1 & 0,5 & 0,6 \\
\hline & PRgBF & 10,8 & 8,5 & 5,4 & 5,3 & 1,0 & 1,2 \\
\hline & PRIrBF & 9,2 & 6,8 & 2,4 & 1,8 & 1,0 & 0,4 \\
\hline & PIR & 20,8 & 17,1 & 13,8 & 9,3 & 4,1 & 9,2 \\
\hline & PIRg & 24,4 & 19,4 & 15,3 & 14,1 & 5,6 & 9,6 \\
\hline & PIIr & 23,4 & 17,4 & 13,0 & 11,5 & 5,2 & 10,0 \\
\hline \multirow{9}{*}{ 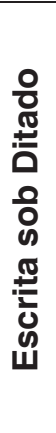 } & PRRAF & 9,8 & 9,0 & 3,0 & 4,1 & 2,0 & 1,5 \\
\hline & PRgAF & 9,6 & 7,1 & 2,4 & 4,0 & 2,0 & 1,0 \\
\hline & PRIrAF & 14,1 & 13,9 & 9,9 & 12,1 & 6,5 & 6,4 \\
\hline & PRRBF & 13,1 & 10,8 & 4,3 & 8,1 & 1,8 & 1,7 \\
\hline & PRgBF & 12,8 & 12,7 & 7,8 & 9,1 & 3,9 & 3,5 \\
\hline & PRIrBF & 11,3 & 9,6 & 3,4 & 5,3 & 2,3 & 2,1 \\
\hline & PIR & 25,4 & 21,8 & 13,4 & 13,0 & 8,1 & 6,0 \\
\hline & PIRg & 28,9 & 24,4 & 24,2 & 26,0 & 19,3 & 17,1 \\
\hline & PIIr & 28,3 & 22,0 & 19,3 & 20,0 & 12,9 & 10,9 \\
\hline
\end{tabular}

Legenda: SiS: Síntese Silábica; SiF: Síntese Fonêmica; Rim: Rima; Alit: Aliteração; SeS: Segmentação Silábica; SeF: Segmentação Fonêmica; ManS: Manipulação Silábica; ManF: Manipulação Fonêmica; TrS: Transposição Silábica; TrF: Transposição Fonêmica; ET: Escore Total; PRRAF: Palavras Reais Regulares de Alta Frequência; PRgAF: Palavras Reais Regra de Alta Frequência; PRIrAF: Palavras Reais Irregulares de Alta Frequência; PRRBF: Palavras Reais Regulares de Baixa Frequência; PRgBF: Palavras Reais Regra de Baixa Frequência; PRIrBF: Palavras Reais Irregulares de Baixa Frequência; PIR: Palavras Inventadas Regulares; PIRg: Palavras Inventadas Regra; PIIr: Palavras Inventadas Irregulares.

Análise estatística: Teste Mann-Whitney.

à rima e à aliteração, os GIII, GIV, GV e GVI não apresentaram estas diferenças. Nos GIV e GV, não houve diferenças nos subtestes de transposição silábica e transposição fonêmica. Também não aconteceram as diferenças para a comparação entre as habilidades de manipulação silábica e manipulação fonêmica para nenhum dos grupos deste estudo (Tabela 4).

$\mathrm{Na}$ comparação intra-grupo das médias referentes ao desempenho dos grupos na leitura oral para a mesma categoria de palavras em relação à frequência de ocorrência é possível verificar que 
Tabela 2 - Distribuição do valor de p referente ao desempenho dos escolares dos GI, GII, GIII, GIV, GV e GVI no RAN, PCF e Leitura Oral e Escrita sob ditado de palavras e pseudo-palavras

\begin{tabular}{|c|c|c|c|c|}
\hline \multirow{5}{*}{ RAN } & Habilidades & GI X GIV & GII X GV & GIII X GVI \\
\hline & Cores & 0,064 & 0,053 & $0,001^{*}$ \\
\hline & Letras & 0,909 & $0,017^{*}$ & $0,001^{*}$ \\
\hline & Números & 0,761 & $0,028^{*}$ & 0,094 \\
\hline & Objetos & 0,150 & $0,004^{*}$ & $0,001^{*}$ \\
\hline \multirow{11}{*}{ PCF } & SiS & $>0,999$ & $>0,999$ & $>0,999$ \\
\hline & SiF & 0,461 & $0,008^{*}$ & $>0,999$ \\
\hline & Rim & 0,198 & $0,005^{*}$ & 0,147 \\
\hline & Alit & $>0,999$ & 0,317 & $>0,999$ \\
\hline & SeS & $>0,999$ & $>0,999$ & $>0,999$ \\
\hline & SeF & 0,077 & 0,087 & 0,751 \\
\hline & ManS & $0,013^{*}$ & 0,068 & $>0,999$ \\
\hline & ManF & $0,016^{*}$ & $0,030^{*}$ & 0,317 \\
\hline & TrS & 0,503 & 0,317 & 0,317 \\
\hline & TrF & 0,874 & $0,005^{*}$ & 0,262 \\
\hline & ET & 0,110 & $0,002^{*}$ & 0,543 \\
\hline \multirow{9}{*}{ Leitura Oral } & PRRBF & 0,542 & 0,053 & $0,004^{*}$ \\
\hline & PRRAF & 0,067 & $0,020^{*}$ & $>0,999$ \\
\hline & PRIrBF & 0,086 & 0,089 & 0,355 \\
\hline & PRIrAF & $0,007^{*}$ & $0,034^{*}$ & 0,053 \\
\hline & PRgBF & $0,009^{*}$ & $0,026^{*}$ & $0,012^{*}$ \\
\hline & PRgAF & $0,002^{*}$ & $0,006^{*}$ & $0,012^{*}$ \\
\hline & PIR & $<0,001^{*}$ & $0,002^{*}$ & $0,019^{*}$ \\
\hline & PIIr & $0,006^{*}$ & $0,002^{*}$ & $<0,001^{*}$ \\
\hline & PIRg & $0,005^{\star}$ & $<0,001^{*}$ & 0,347 \\
\hline \multirow{9}{*}{$\begin{array}{l}\text { Escrita sob } \\
\text { Ditado }\end{array}$} & PRRBF & $0,015^{*}$ & 0,638 & $0,003^{*}$ \\
\hline & PRRAF & $0,025^{*}$ & $<0,001^{*}$ & $<0,001^{*}$ \\
\hline & PRIrBF & $0,035^{*}$ & $0,012^{*}$ & $0,002^{*}$ \\
\hline & PRIrAF & $0,005^{\star}$ & 0,126 & $<0,001^{*}$ \\
\hline & PRgBF & 0,053 & $<0,001^{*}$ & $<0,001^{*}$ \\
\hline & PRgAF & $0,014^{*}$ & $0,002^{*}$ & 0,240 \\
\hline & PIR & $0,004^{*}$ & $0,001^{*}$ & $0,004^{*}$ \\
\hline & PIIr & $0,009^{*}$ & 0,095 & 0,360 \\
\hline & PIRg & $0,005^{*}$ & $<0,001^{*}$ & 0,347 \\
\hline
\end{tabular}

Legenda: SiS: Síntese Silábica; SiF: Síntese Fonêmica; Rim: Rima; Alit: Aliteração; SeS: Segmentação Silábica; SeF: Segmentação Fonêmica; ManS: Manipulação Silábica; ManF: Manipulação Fonêmica; TrS: Transposição Silábica; TrF: Transposição Fonêmica; ET: Escore Total; PRRAF: Palavras Reais Regulares de Alta Frequência; PRgAF: Palavras Reais Regra de Alta Frequência; PRIrAF: Palavras Reais Irregulares de Alta Frequência; PRRBF: Palavras Reais Regulares de Baixa Frequência; PRgBF: Palavras Reais Regra de Baixa Frequência; PRIrBF: Palavras Reais Irregulares de Baixa Frequência; PIR: Palavras Inventadas Regulares; PIRg: Palavras Inventadas Regra; PIIr: Palavras Inventadas Irregulares.

Análise estatística: Teste Mann-Whitney.

o GIII apresentou diferenças estatisticamente significantes para leitura de palavras reais regulares, enquanto o $\mathrm{Gl}$ apresentou esta diferença para palavras reais irregulares e os demais grupos não apresentaram esta diferença em nenhum subteste de leitura, como podemos perceber na Tabela 5.
Na comparação intra-grupo das médias referentes ao desempenho dos grupos quanto à leitura oral da categoria de palavras inventadas é possível observar diferenças estatisticamente significantes para os GIII e GV quando comparadas à leitura oral de palavras inventadas regulares e irregula- 
Tabela 3 - Distribuição do valor de p referente ao desempenho dos escolares dos GI, GII, GIII, GIV, GV e GVI quanto aos sub testes de cores, letras, números e objetos do Teste de Nomeação Rápida Automática (RAN)

\begin{tabular}{|c|c|c|c|c|c|c|}
\hline \multirow{2}{*}{ Subtestes } & \multicolumn{6}{|c|}{ Grupos } \\
\hline & $\mathbf{I}$ & II & III & IV & $\mathbf{V}$ & VI \\
\hline Cores X Letras & $0,005^{\star}$ & $0,005^{\star}$ & $0,005^{*}$ & $0,005^{\star}$ & $0,005^{*}$ & $0,005^{*}$ \\
\hline Cores X Números & $0,005^{\star}$ & $0,005^{*}$ & $0,005^{*}$ & $0,005^{\star}$ & $0,005^{*}$ & $0,037^{*}$ \\
\hline Cores X Objetos & $0,009^{*}$ & $0,005^{*}$ & $0,005^{*}$ & 0,114 & $0,024^{*}$ & $0,008^{*}$ \\
\hline Letras X Números & 0,714 & 0,201 & $0,015^{*}$ & 0,610 & 0,165 & $0,028^{*}$ \\
\hline Letras X Objetos & $0,005^{\star}$ & $0,005^{*}$ & $0,005^{*}$ & $0,005^{\star}$ & $0,005^{*}$ & $0,005^{*}$ \\
\hline Números X Objetos & $0,005^{*}$ & $0,005^{*}$ & $0,005^{*}$ & $0,005^{\star}$ & $0,005^{*}$ & $0,014^{*}$ \\
\hline
\end{tabular}

Análise estatística: Teste Wilcoxon.

Tabela 4 - Distribuição do valor de p referente ao desempenho dos escolares dos GI, GII, GIII, GIV, GV e GVI quanto às habilidades fonêmicas, supra-fonêmicas e silábicas na PCF

\begin{tabular}{ccccccc}
\hline \multirow{2}{*}{ Subtestes } & \multicolumn{7}{c}{ Grupos } \\
\cline { 2 - 7 } & $\mathbf{I}$ & II & III & IV & V & VI \\
\hline SiS x SiF & $0,007^{*}$ & $0,006^{*}$ & $0,020^{*}$ & $0,010^{*}$ & $0,014^{*}$ & $0,026^{*}$ \\
Rim x Alit & $0,023^{*}$ & $0,024^{*}$ & 0,180 & $>0,999$ & $>0,999$ & $>0,999$ \\
SeS x SeF & $0,004^{*}$ & $0,007^{*}$ & $0,005^{*}$ & $0,026^{*}$ & $0,011^{*}$ & $0,004^{*}$ \\
ManS x ManF & 0,414 & 0,317 & 0,317 & 0,317 & $>0,999$ & $>0,999$ \\
TrS x TrF & $0,026^{*}$ & $0,037^{*}$ & $0,024^{*}$ & 0,102 & 0,317 & $0,039^{*}$ \\
\hline
\end{tabular}

Legenda: SiS: Síntese Silábica; SiF: Síntese Fonêmica; Rim: Rima; Alit: Aliteração; SeS: Segmentação Silábica; SeF: Segmentação Fonêmica; ManS: Manipulação Silábica; ManF: Manipulação Fonêmica; TrS: Transposição Silábica; TrF: Transposição Fonêmica. Análise estatística: Teste Wilcoxon.

Tabela 5 - Distribuição do valor de p referente ao desempenho dos escolares dos GI, GIl, GIII, GIV, GV e GVI quanto à leitura de palavras reais

\begin{tabular}{ccccccc}
\hline \multirow{2}{*}{ Categoria de palavras } & \multicolumn{7}{c}{ Grupos } \\
\cline { 2 - 7 } & I & II & III & IV & V & VI \\
\hline PRRAF - PRRBF & 0,157 & $>0,999$ & $0,010^{*}$ & 0,317 & $>0,999$ & 0,157 \\
PRIrAF - PRIrBF & $0,039^{*}$ & 0,058 & 0,582 & 0,066 & 0,059 & 0,180 \\
PRgAF - PRgBF & 0,132 & 0,268 & $>0,999$ & 0,083 & 0,102 & $>0,999$ \\
\hline
\end{tabular}

Legenda: PRRAF: Palavras Reais Regulares de Alta Frequência; PRgAF: Palavras Reais Regra de Alta Frequência; PRIrAF: Palavras Reais Irregulares de Alta Frequência; PRRBF: Palavras Reais Regulares de Baixa Frequência; PRgBF: Palavras Reais Regra de Baixa Frequência; PRIrBF: Palavras Reais Irregulares de Baixa Frequência.

Análise estatística: Teste Wilcoxon.

Tabela 6 - Distribuição do valor de p referente ao desempenho dos escolares dos GI, GII, GIII, GIV, GV e GVI quanto à leitura de palavras inventadas

\begin{tabular}{ccccccc}
\hline \multirow{2}{*}{ Categoria de palavras } & \multicolumn{7}{c}{ Grupos } \\
\cline { 2 - 7 } & I & II & III & IV & V & VI \\
\hline PIR x PIIr & 0,396 & - & $0,009^{*}$ & - & $0,017^{*}$ & 0,726 \\
PIR x PIRg & $0,007^{*}$ & - & 0,441 & - & 0,340 & 0,107 \\
PIIr x PIRg & $0,015^{*}$ & - & $0,005^{\star}$ & - & $0,041^{*}$ & $0,041^{*}$ \\
\hline
\end{tabular}

Legenda: PIR: Palavras Inventadas Regulares; PIRg: Palavras Inventadas Regra; PIIr: Palavras Inventadas Irregulares.

Análise estatística: Teste Wilcoxon. 
Tabela 7 - Distribuição do valor de p referente ao desempenho dos escolares dos GI, GII, GIII, GIV, GV e GVI quanto à escrita de palavras reais

\begin{tabular}{ccccccc}
\hline \multirow{2}{*}{ Categoria de palavras } & \multicolumn{7}{c}{ Grupos } \\
\cline { 2 - 7 } & $\mathbf{I}$ & II & III & IV & V & VI \\
\hline PRRAF - PRRBF & 0,732 & 0,141 & $0,020^{*}$ & 0,380 & $0,020^{*}$ & 0,763 \\
PRIrAF - PRiRBF & $0,008^{*}$ & $0,005^{*}$ & $0,004^{*}$ & $0,005^{*}$ & $0,029^{*}$ & $0,161^{*}$ \\
PRgAF - PRgBF & $0,011^{*}$ & $0,011^{*}$ & $0,005^{*}$ & $0,009^{*}$ & $>0,999$ & 0,234 \\
\hline
\end{tabular}

Legenda: PRRAF: Palavras Reais Regulares de Alta Frequência; PRgAF: Palavras Reais Regra de Alta Frequência; PRIrAF: Palavras Reais Irregulares de Alta Frequência; PRRBF: Palavras Reais Regulares de Baixa Frequência; PRgBF: Palavras Reais Regra de Baixa Frequência; PRIrBF: Palavras Reais Irregulares de Baixa Frequência.

Análise estatística: Teste Wilcoxon.

Tabela 8 - Distribuição do valor de p referente ao desempenho dos escolares dos GI, GIl, GIII, GIV, GV e GVI quanto à escrita de palavras inventadas

\begin{tabular}{ccccccc}
\hline \multirow{2}{*}{ Categoria de palavras } & \multicolumn{7}{c}{ Grupo } \\
\cline { 2 - 7 } & I & II & III & IV & V & VI \\
\hline PIR x PIIr & $0,005^{\star}$ & $0,005^{*}$ & $0,015^{\star}$ & $0,005^{\star}$ & $0,005^{\star}$ & $0,005^{\star}$ \\
PIR x PIRg & $0,009^{\star}$ & $0,004^{*}$ & 0,063 & $0,008^{\star}$ & $0,018^{*}$ & $0,011^{\star}$ \\
PIIr x PIRg & $0,005^{\star}$ & $0,005^{\star}$ & 0,066 & $0,008^{\star}$ & $0,005^{\star}$ & $0,034^{\star}$ \\
\hline
\end{tabular}

Legenda: PIR: Palavras Inventadas Regulares; PIRg: Palavras Inventadas Regra; PIIr: Palavras Inventadas Irregulares. Análise estatística: Teste Wilcoxon.

res, para o Gl quando comparadas à leitura oral de palavras inventadas regulares e regra e, para os $\mathrm{Gl}$, GIII, GV e GVI quando comparadas à leitura oral de palavras inventadas irregulares e regra, conforme dados apresentados naTabela 6 .

Quando realizada a comparação intra-grupo das médias referentes ao desempenho dos grupos na escrita de palavras de mesma categoria em relação à frequência de ocorrência é possível observar que os grupos GIII e GV apresentaram diferenças estatisticamente significante nas palavras reais regulares, enquanto os GI, GII, GIII e GIV apresentaram estas diferenças para palavras reais regra, como mostra a Tabela 7.

Com a comparação intra-grupo das médias referentes ao desempenho dos grupos quanto à escrita da categoria de palavras inventadas é possível verificar diferenças estatisticamente significantes para todos os grupos quando comparados à escrita de palavras inventadas regulares e irregulares, como também para os GI, GIl, GIV, GV, e GVI quando comparados à escrita de palavras inventadas regulares e regra e palavras inventadas irregulares e regra (Tabela 8).

\section{DISCUSSÃO}

Para a aprendizagem do sistema alfabético de escrita, é necessária a associação de um compo- nente auditivo fonêmico a um componente visual gráfico. Para a compreensão do princípio alfabético, são necessários três fatores: a ciência de que é possível segmentar a língua falada em unidades distintas; o entendimento de que essas mesmas unidades repetem-se em diferentes palavras faladas e a noção das regras de correspondência entre grafemas e fonemas. Destaca-se que os dois primeiros fatores são aspectos da consciência fonológica, e isto a coloca como indispensável no desenvolvimento da leitura e escrita ${ }^{19,20}$.

A percepção da estrutura fonêmica da fala permite que a criança utilize um sistema gerativo para converter ortografia em fonologia, permitindo, assim, a leitura de qualquer palavra regular que envolva a correspondência grafo-fonêmica. A característica gerativa das ortografias alfabéticas permite aos leitores aprenderem por si mesmos, uma vez que, ao encontrar palavras novas, eles podem aplicar as regras de decodificação fonológica. O processo de decodificação fonológica contribui para que a criança forme a representação ortográfica da nova palavra, portanto, é o processo fonológico que permitirá à criança, posteriormente, realizar leitura pela rota lexical ${ }^{21-23}$

O processamento fonológico refere-se às operações de processamento de informação baseadas na estrutura fonológica da linguagem oral e envolve a percepção e a memória de trabalho ${ }^{8-12}$. Quando 
acontecem falhas no processamento fonológico da informação, a análise da palavra articulada em suas partes constituintes, sílabas e sons fica prejudicada em decorrência da presença de desvios na representação fonética na memória de curto prazo, influenciando negativamente nos aspectos de produção da fala e a produção oral do texto lido ${ }^{21,24,25}$.

Os achados deste estudo revelaram que os escolares com dificuldades de aprendizagem, independentemente da seriação, apresentaram obstáculos em acessar e recuperar informações fonológicas necessárias para o bom desempenho em tarefas de leitura oral e escrita por meio de ditado de palavras, conforme referido pela literatura e citado anteriormente.

Em relação ao desempenho dos escolares no RAN, os escolares dos grupos sem dificuldades foram melhores do que os dos grupos com dificuldades de aprendizagem. Neste estudo, observou-se que os escolares dos GIV, GV e GVI apresentaram melhor desempenho para nomeação de cores em relação a objetos e, de um modo geral, apresentaram maior velocidade para nomeação de letras em relação a cores, de números em relação a cores, de letras em relação a objetos e de números em relação a objetos. Foi possível observar ainda a nomeação mais rápida para letras e números do que para cores, uma vez que a nomeação de estímulos requer uso de processos atencionais, perceptivos e visuais para recuperação de léxico de maior extensão, corroborando com os achados ${ }^{15,16,26,27}$. Em relação ao desempenho dos escolares na PCF, verificou-se que os escolares de todos os grupos apresentaram melhores desempenhos nas habilidades silábicas do que nas habilidades fonêmicas; mais especificamente, melhores resultados em segmentação e transposição silábicas do que fonêmicas.

Em rima e aliteração, nos GIII, GIV, GV e GVI, não ocorreram diferenças estatisticamente significantes. Somente os Gl e Gll, com dificuldades de aprendizagem, apresentaram estas habilidades alteradas, o que permite considerar que esses escolares apresentam falhas na percepção dos sons iniciais e finais das palavras, evidenciando dificuldades de agrupamento de palavras que apresentam similaridade fonológica, revelando contratempos no uso da memória de trabalho fonológico, o que compromete a formação de novas palavras a partir de segmento silábico e fonêmico adquirido no início da alfabetização ${ }^{13,28}$.

Pesquisadores concordam que a habilidade fonológica é importante para a aquisição da leitura e que, na maioria dos indivíduos com atraso em leitura ou dislexia, identificam-se alterações nessa habilidade. Esta hipótese tem sido sustentada por inúmeros trabalhos que demonstram atrasos na sensibilidade à rima, à aliteração e à segmentação fonêmica durante o desenvolvimento da leitura ${ }^{29-32}$.

Verificou-se ainda, que os escolares apresentaram melhor desempenho em leitura oral e escrita por meio de ditado de palavras reais do que de palavras inventadas, nas de alta frequência do que nas de baixa frequência e em palavras inventadas regulares do que em regra e irregulares, corroborando estudo anterior ${ }^{33}$, que afirmaram que quanto mais a criança tem contato perceptivo, auditivo e visual com as palavras, mais estas palavras se tornam familiares. Dessa forma, a criança lerá melhor as palavras de alta frequência do que as de baixa frequência e as palavras reais mais do que as inventadas. Nas comparações feitas de palavras reais de alta frequência regulares, irregulares e regra, somente os GI e GIII apresentaram diferenças estatisticamente significantes, uma vez que os escolares destes grupos são crianças com dificuldades de aprendizagem, mostrando melhor desempenho para leitura de palavras de alta frequência do que para palavras de baixa frequência.

Nas comparações entre as palavras inventadas regulares, irregulares e regra, somente os Grupos GII e GIV não apresentaram diferenças estatisticamente significantes. $\mathrm{O}$ fato dos escolares deste estudo apresentarem maiores dificuldades na leitura de palavras inventadas nos permite considerar que aqueles que apresentam dificuldades na percepção da estrutura fonológica das palavras quando estas já estão no seu léxico de input visual, apresentam dificuldade de analisar fonológica e ortograficamente a palavra durante a leitura ou escrita ${ }^{34,35}$.

Em relação ao desempenho dos escolares na escrita por meio de ditado, observa-se que, como na prova de leitura oral, o desempenho é maior para escrita de palavras reais de alta frequência do que de palavras de baixa frequência, de palavras inventadas regulares do que irregulares e regra, conforme descrito na literatura ${ }^{32}$ que afirmou que o léxico de alta frequência é arquivado no léxico grafêmico, facilitando, assim, o desenvolvimento de uma escrita sem erros.

Os achados deste estudo permitem verificar a relação entre a velocidade e tempo de nomeação, ou seja, acesso ao léxico mental com habilidades fonológicas e a leitura e escrita. Os GIV, GV e GVI, de escolares sem dificuldades de aprendizagem, apresentaram maior velocidade de nomeação em menor tempo e, portanto, melhor desempenho fonológico e maiores habilidades de leitura e escrita por meio de ditado de palavras isoladas. Já os GI, Gll e GIII apresentaram menor velocidade de nomeação em um espaço maior de tempo, consequentemente, apresentaram desempenho inferior nas provas de 
consciência fonológica, leitura e escrita por meio de ditado de palavras isoladas. Esses achados confirmam os resultados descritos na literatura ${ }^{26}$.

Essas descobertas evidenciaram que a velocidade de acesso ao léxico mental está diretamente relacionada com a habilidade de consciência fonológica e de leitura e escrita, pois os escolares dos grupos sem dificuldades de aprendizagem apresentaram menor relação velocidade/tempo em tarefas de nomeação e, assim, melhor desempenho em consciência fonológica e em atividade de leitura oral e escrita por meio de ditado de palavras isoladas, em comparação ao desempenho dos grupos com dificuldades de aprendizagem que apresentaram maior relação velocidade/tempo em tarefas de nomeação e, consequentemente, desempenho inferior em tarefas de consciência fonológica e da leitura e escrita de palavras isoladas.

As relações estabelecidas neste estudo entre velocidade de nomeação e automatização dos estímulos apresentados com a capacidade de acesso lexical, discriminação visual, frequência de uso dos estímulos e competição para a apresentação no menor tempo possível para a nomeação dos códigos são necessárias para o estabelecimento do mecanismo de conversão fonema-grafema exigido na realização da leitura e escrita de um sistema de escrita com base alfabética como o português.

Os resultados deste estudo evidenciaram a relação entre o RAN, a habilidade fonológica, a leitura e a escrita, pois os discentes com dificuldades de aprendizagem apresentaram velocidade de nomeação e habilidade fonológica alteradas, o que refletiu diretamente no desempenho inferior dos mesmos em leitura e escrita de palavras ${ }^{36,37}$.

No Brasil, o conhecimento do perfil de escolares com dificuldade de aprendizagem é de fundamental importância, pois a alta proporção de escolares que apresentam problemas em habilidades fonológicas e nomeação rápida os fazem ser confundidos com crianças que apresentam o quadro de dislexia do desenvolvimento, quando, na verdade, o que apresentam é uma falha no acesso fonológico da informação decorrente de problemas de alfabetização. A busca pelo estabelecimento do perfil tanto de escolares com dificuldades de aprendizagem como o de escolares com dislexia do desenvolvimento pode contribuir para melhor compreensão da influência do meio acadêmico sobre a aprendizagem da criança. Neste estudo, verificou-se que os escolares com dificuldades de aprendizagem apresentaram melhores desempenhos nas medidas empregadas do que em estudos da literatura nacional com escolares disléxicos ${ }^{7,27}$.
Desta forma, há necessidade de aprofundamento dos estudos fonoaudiológicos na investigação de habilidades fonológicas e de nomeação rápida em âmbito escolar, uma vez que a literatura internacional considera as mesmas habilidades preditoras do bom desempenho em leitura e escrita de um sistema de escrita com base alfabética ${ }^{26,32}$. Assim, essa análise poderia contribuir para o preenchimento de uma lacuna existente ainda na fonoaudiologia, que é o estabelecimento do perfil do mau leitor e do leitor disléxico, o que evitaria a confusão diagnóstica e suas consequências, que geralmente afetam a qualidade de vida das crianças no contexto familiar, social e educacional.

\section{CONCLUSÃO}

Os resultados deste estudo permitem concluir que os escolares dos GIV, GV e GVI apresentaram melhores desempenhos em consciência fonológica, nomeação rápida, leitura e escrita de palavras isoladas ao longo da seriação, evidenciando a melhora nas habilidades de leitura e escrita com a exposição às atividades de sala de aula. Entretanto, o mesmo não foi evidenciado entre os escolares dos GI, GIl e GIII, que não apresentaram melhora em desempenho nas avaliações e provas aplicadas nesta pesquisa, indicando que, quando presentes, as dificuldades de aprendizagem não são superadas ao longo da seriação escolar, desencadeando ao longo do processo de alfabetização comprometimento quanto ao desempenho acadêmico.

Os escolares dos GI, Gll e GIII, com problemas de aprendizagem apresentaram maior relação velocidade/tempo em tarefas de nomeação e, consequentemente, desempenho inferior em tarefas de consciência fonológica e leitura e escrita de palavras isoladas quando comparados aos escolares dos GIV, GV e GVI, sem dificuldades de aprendizagem; revelando, assim, comprometimento na relação entre as capacidades de nomeação e automatização dos estímulos apresentados, com a capacidade de acesso lexical, discriminação visual, frequência de uso dos estímulos e competição para a apresentação do menor tempo possível para a nomeação dos códigos, necessários no estabelecimento do mecanismo de conversão fonema-grafema, exigido para a realização da leitura e escrita em um sistema gráfico e alfabético como se dá na língua portuguesa. 


\begin{abstract}
Purpose: characterizing and comparing the performance of students with and without learning difficulties from the private education in phonological awareness, rapid naming, reading and writing. Methods: sixty private students from $2^{\text {nd }}$ to $4^{\text {th }}$ grade participated, distributed into 6 groups - each one was composed of 10 students being 3 groups of students with learning difficulties and 3 groups without such difficulties. As procedure was used the phonological awareness, rapid automatized naming and oral reading and writing under dictation. Results: the results highlighted a superior development of those who without learning difficulties compared to the students with learning difficulties. Conclusion: the students with learning difficulties presented a higher score in the time/speed task of rapid naming and, consequently, lower production in activities of phonological awareness and reading and writing, when compared to students without difficulties. Thus, a relation engagement between rapid naming and lexical access is revealed, as well as between visual discrimination, frequency of word occurrence and presentation of the code naming needed to do the phoneme-grapheme conversion process required in the reading and writing in an alphabetic writing system such as the Portuguese language.
\end{abstract}

KEYWORDS: Reading; Learning Disorders; Education

\section{REFERÊNCIAS}

1. Rebello JAS. Dificuldades da leitura e da escrita em alunos do ensino básico. Portugal: Edições Asa; 1993. p. 69-136.

2. Smith C, Strick L. Dificuldades de aprendizagem de A a Z: um guia completo para pais e educadores. Porto Alegre: Artes Médicas; 2001. p. 13-98.

3. Pilati O. Sistema nacional de avaliação da educação básica (SAEB): ensaio. Avaliação das Políticas Públicas Educacionais. 1994; 2:11-30.

4. Brasil. Ministério da Educação. Repetência é fator de impacto na queda do rendimento escolar. Brasília, DF: INEP - Instituto Nacional de Estudos e Pesquisas Educacionais 2002. Disponível em: URL: http://www.inep.gov.br. Acesso em 17 set 2005.

5. Brasil. Ministério da Educação. Resultados do SAEB-2003 - versão preliminar. Brasília, DF: INEP - Instituto Nacional de Estudos e Pesquisas Educacionais; 2004.

6. Ciasca SM, Capellini SA, Tonelotto JMF. Distúrbios específicos de aprendizagem. In: Ciasca SM, organizador. Distúrbio de aprendizagem: proposta de avaliação interdisciplinar. São Paulo: Casa do Psicólogo; 2003. p. 55-66.

7. Capellini AS, Tonelotto JMF, Ciasca SM. Medidas de desempenho escolar: avaliação formal e opinião de professores. Rev Est Psicol. 2004; 21(2):79-90.

8. Wagner RK, Torgensen JK, Rashotte CA, Hecht SA, Barker TA, Burgess SR, et al. Changing relations between phonological processing abilities and wordlevel reading as children develop from beginning to skilled readers: a 5 year longitudinal study. Develop Psychol. 1997; 33(3):468-79.
9. Scheltinga $F$, Van Der Leij A, Van Beinun F. Importance of phonological skills and underlying processes to reading achievement: a study on dyslexic and specific language impaired children. IFA Proc. 2003; (25):21-30.

10. Cardoso-Martins C, Pennington BF. Qual é a contribuição da nomeação seriada rápida para a habilidade de leitura e escrita? Evidência de crianças e adolescentes com e sem dificuldade de leitura. Psicol Reflex Crit. 2004; 14(2):387-97.

11. Sprugevica I, Hoien T. Early phonological skills as a predictor of reading acquisition: a follow-up study from kindergarten to the middle of grade 2 . Scand J Psychol. 2003; 44(2):119-24.

12. Vukovic RK, Siegel LS. The double-deficit hypothesis: a comprehensive analysis of the evidence. J Learn Disabil. 2006; 39(1):25-47.

13. Van der Leij A, Morfidi E. Core deficits and variable differences in Dutch poor readers learning English. J Learn Disabil. 2006; 39(1):74-90.

14. Ávila CRB, Capellini SA. Relation between oral and written language. In: Capellini SA. Neuropsycholinguistic perspectives on dyslexia and other learning disabilities. New York: New Science Publisher; 2007. p.15-22.

15. Denckla MB, Rudel R. Rapid automatized naming of picture objects, colors, letters, and numbers by normal children. Cortex. 1974; 10(1):186-202.

16. Ferreira TL, Capellini SA, Ciasca SM, Tonelotto JMF. Desempenho de escolares leitores proficientes no teste de nomeação automatizada rápida RAN. Temas Desenvolv. 2003; 12(69):26-32.

17. Capovilla AGS, Capovilla FC. Prova de consciência fonológica: desenvolvimento de dez 
habilidades da pré-escola à segunda série. Temas Desenvolv. 1998; 7(37):14-20.

18. Pinheiro AMV. Leitura e escrita: uma abordagem cognitiva. Campinas: Psy II, 1994. p. 131-41.

19. Barrera SD, Maluf MR. Consciência metalinguística e alfabetização: um estudo com crianças da primeira série do ensino fundamental. Psicol Reflex Crít. 2003; 3(16):491-502.

20. Guimarães SRK. Dificuldades no desenvolvimento da lectoescrita: o papel das habilidades metalinguísticas. Psicol Teor Pesq. 2003; 19(1): 33-45.

21. Capellini SA. Dificuldade escolar e distúrbio de aprendizagem. In: Ribeiro do Valle LE. Temas multidisciplinares em neuropsicologia e aprendizagem. São Paulo: Robe Editorial; 2004. p. 465-74.

22. Capellini SA. Distúrbios de aprendizagem versus dislexia. In: Ferreira L, Befi-Lopes D, Limongi S. Tratado de fonoaudiologia. São Paulo: Roca; 2004. $1058 p$.

23. Veuillet E, Magnan A, Ecalle J, Thain-Van H, Collet L. Auditory processing disorder in children with reading disabilities: effect of audiovisual training. Brain. 2007; 130(11):2915-28.

24. Salgado CA, Capellini SA. Desempenho em leitura e escrita de escolares com transtorno fonológico. Psicol Esc Educ. 2004; 8(2):179-88.

25. Barbosa T. Memória operacional fonológica, consciência fonológica e linguagem nas dificuldades de alfabetização. [dissertação]. São Paulo (SP): Universidade Federal de São Paulo; 2005.

26. Swanson HL, Howard CB, Saez L. Do different components of working memory underlie different subgroups of reading disabilities? J Learn Disab. 2006; 39(3):252-69.

27. Capellini SA, Ferreira TL, Salgado CA, Ciasca SM. Desempenho de escolares bons leitores, com dislexia e com transtorno do déficit de atenção e hiperatividade em nomeação automática rápida. Rev Soc Bras Fonoaudiol. 2007; 12(2):114-9.
28. Steinbrink C, Klatte M. Phonological working memory in German children with poor reading and spelling abilities. Dyslexia. 2008; 14(4):271-90.

29. Wolf M, Bowers PG, Biddle K. Naming-speed processes, timing, and reading: a conceptual review. J Learn Disabil. 2000; 33(4):387-407.

30. Bowers PG, Newby-Clark E. The role of naming speed within a model of reading acquisition. Read Writ: Interd J. 2002; 15(1-2):109-26.

31. Vukovic RK, Wilson AM, Nash KK. Naming speed deficits in adults with reading disabilities: a test of the double-deficit hypothesis. J Learn Disabil. 2004; 37(5):440-50.

32. Savege RS, Frederickson N, Goodwin R, Patni U, Smith N, Tuersley L. Relationships among rapid digit naming, phonological processing, motor automaticity, and speech perception in poor, average, and good readers and spellers. J Learn Disabil. 2005; 38(1):12-28.

33. Snowling MJ, Gallagher A, Frith U. Family risk of dyslexia is continuous: individual differences in the precursors of reading skill. Child Dev. 2003; 74(2):358-73.

34. Snowling M, Stackhouse J. Dislexia, fala e linguagem: um manual do profissional. Porto Alegre: Artes Médicas; 2004. p. 57-121.

35. Hogan TP, Catts HW, Little TD. The relationship between phonological awareness and reading: implications for the assessment of phonological awareness. Lang Speech Hear Serv Sch. 2005; 36(4):285-93.

36. Van Bon WHJ, Bouwmans M, Broeders INLDC. The prevalence of poor reading in Dutch special elementary education. J Learn Disabil. 2006; 39(6):482-95.

37. Protopapas A, Skaloumbakas C. Traditional and computer-based screening and diagnosis of reading disabilities in Greek. J Learn Disabil. 2007; 40(1):15-36.

DOI: 10.1590 / S1516-18462009005000002

RECEBIDO EM: 13/12/2007

ACEITO EM: 25/08/2008

Endereço para correspondência:

Simone Aparecida Capellini

Rua Bartolomeu de Gusmão, 10-84

Bauru - SP

CEP: 17017-326

E-mail: sacap@uol.com.br 